\title{
Functional Fitness and Physical Activity of Portuguese Community-Residing Older Adults
}

\author{
Élvio R. Gouveia, José A. Maia, Gaston P. Beunen, \\ Cameron J. Blimkie, Ercília M. Fena, and Duarte L. Freitas
}

\begin{abstract}
The purposes of this study were to generate functional-fitness norms for Portuguese older adults, to determine age and sex differences, and to analyze the physical activity-associated variation in functional fitness. The sample was composed of 802 older adults, 401 men and 401 women, age 60-79 yr. Functional fitness was assessed using the Senior Fitness Test. Physical activity level was estimated via the Baecke questionnaire. The P50 values decreased from 60 to 64 to 75 to $79 \mathrm{yr}$ of age. A significant main effect for age group was found in all functional-fitness tests. Men scored significantly better than women in the chair stand, 8-ft up-and-go, and 6-min walk. Women scored significantly better than men in chair sit-and-reach and back scratch. Active participants scored better in functional-fitness tests than their average and nonactive peers. This study showed a decline in functional fitness with age, better performance of men, and increased proficiency in active participants.
\end{abstract}

Keywords: aging, Senior Fitness Test, reference data

Portugal, like other countries of southern Europe, has recently experienced a shift toward an increasingly elderly population as a result of low birth rates and increased life expectancy. In 2009, and according to the World Health Organization (2011), life expectancy at birth in Portugal was 76.0 years for men and 82.0 years for women. The proportion of Portuguese people age 65 years or more doubled over the past 40 years, from $8 \%$ in 1960 to $16 \%$ in 2001, and this percentage will double in the next 40 years, to $32 \%$ of the total population in 2050 (Carrilho \& Gonçalves, 2004).

The aging process leads to profound changes in the cardiopulmonary, musculoskeletal, nervous, and immune systems. At rest, stroke volume decreases about $30 \%$ from 25 to 85 years of age. Similarly, there is a progressive reduction of ventilatory peak flow and lung capacity with advancing age (Taylor \& Johnson, 2008). Aging is also characterized by increased bone loss resulting in reduced bone

Gouveia, Fena, and Freitas are with the Dept. of Physical Education and Sports, University of Madeira, Funchal, Portugal. Maia is with the Faculty of Sport, University of Porto, Porto, Portugal. Beunen is with the Faculty of Kinesiology and Rehabilitation Sciences, Katholieke Universiteit Leuven, Leuven, Belgium. Blimkie is with the Dept. of Kinesiology, McMaster University, Hamilton, ON, Canada. 
mineral density. About $25 \%$ of muscle function, defined as the highest lifetime force-generating capacity, is lost by around 65 years of age. Negative changes of the central nervous system are observed in neurotransmitters, nerve conduction, and fine functional-fitness control, and resistance to infectious agents decreases by about 5-30\% through the life span (Spirduso, Francis, \& MacRae, 2005; Taylor \& Johnson, 2008).

The net effect of these changes is a decrease in autonomy and quality of life of older adults. Although environmental and genetic forces may partially explain this decline, changes in physical activity level may also play a prominent role. The benefits of physical activity or exercise are reflected in improved cardiovascular and respiratory functions, reduction of risk factors of coronary heart diseases, and decreased morbidity and mortality (American College of Sports Medicine, 2006).

Rikli and Jones (1999b) published normative functional-fitness scores for older adults age 60-94 in the United States. Results revealed a consistent pattern of decline in performance on all test variables for both men and women over the 5 -year age groups. Performance on muscle-strength and aerobic-endurance tests declines between $32.0 \%$ and $44.0 \%$ from one's early 60 s to early $90 \mathrm{~s}$. Although the pattern of decrease over age was similar in men and women, men scored better on muscle strength, aerobic endurance, and agility/balance; women scored better on flexibility (see Rikli \& Jones, 1999b, for additional details).

In Portugal, very little is known about the functional fitness of older adults. Santos et al. (2008) described the physical activity levels and functional-fitness characteristics of 271 participants, 190 women and 81 men age 65-90 years, from the Autonomous Region of Azores. In brief, older adults walked, on average, $3.8 \pm$ $2.2 \mathrm{~km} /$ day, the large majority of participants fell in the normal (25th-75th percentile) or below-average (below the 25 th percentile) range of scores in the functionalfitness tests, and active men were more physically fit than their nonactive peers.

The assessment of functional fitness in the Autonomous Region of Madeira (ARM) will provide information about normal variation within and across age groups and a database for subsequent research purposes. To our knowledge, this is the first study specifically designed to establish functional-fitness norms for older adults from Portugal. Therefore, the central purposes of the study were threefold: to generate functional-fitness norms for older adults living in the ARM, Portugal; to identify age and sex differences; and to analyze physical activity-associated variation in functional fitness.

\section{Methods}

\section{Sample}

Participants are part of a research project titled Health and Quality of Life of Older Adults in ARM, Portugal. In total, 802 participants (401 men and 401 women) distributed equally over four age cohorts (60-64, 65-69, 70-74, and 75-79 years) were assessed in 2008-09. This study used a cross-sectional design. The demographic and clinical characteristics of participants are shown in Table 1.

The sampling criteria were as follows: geographic area in the 11 districts of ARM, age, functional independence, and no medical contraindications to submaximum exercise according to the guidelines of the American College of Sports 


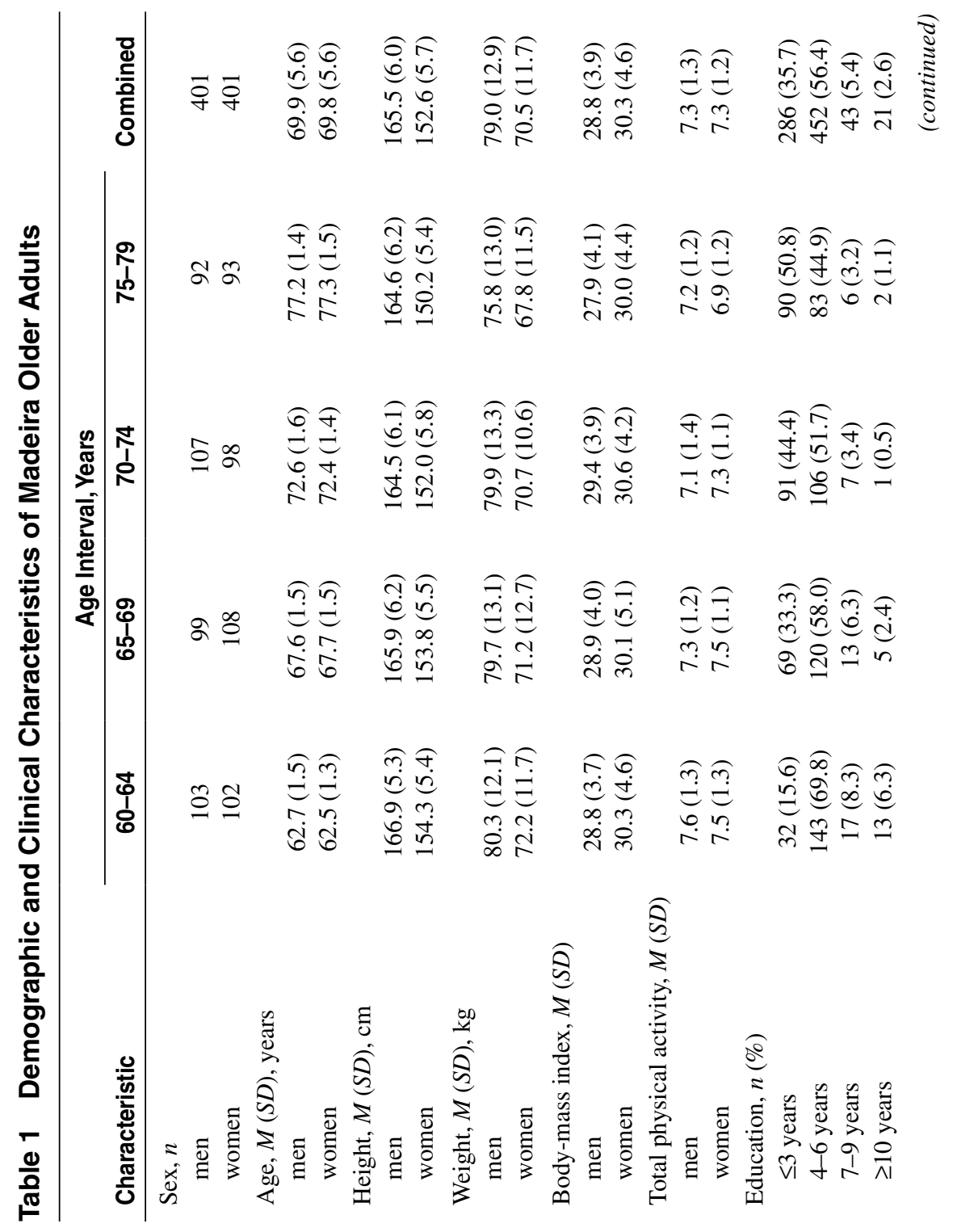




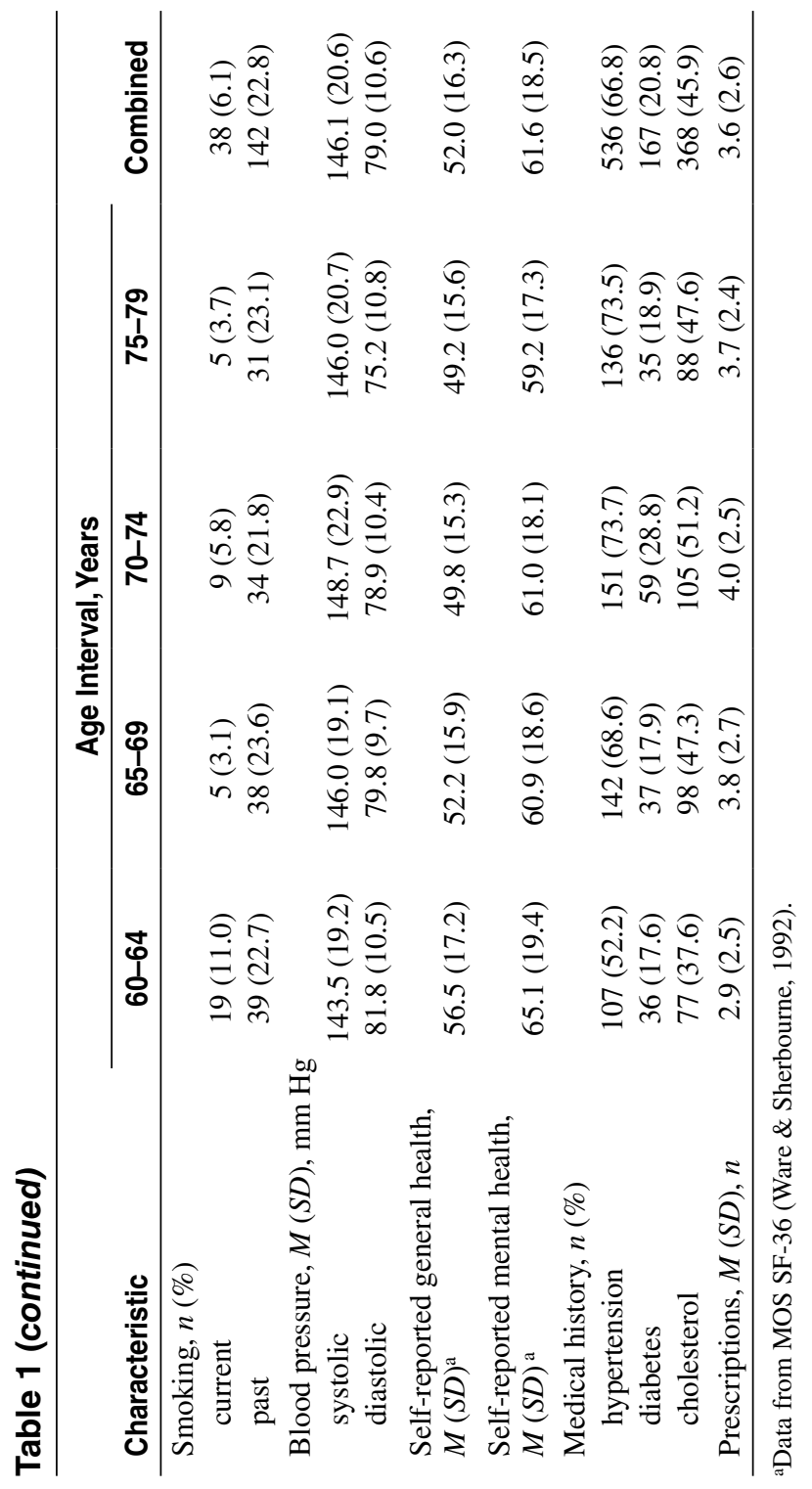


Medicine (2006). In each birth cohort, approximately 100 men and 100 women were included in the sample based on geographic proportional representation; that is, we used samples weighted by age and sex from each district. The proportionate stratified sampling procedure was based on information gathered from the Portuguese census 2001 (Instituto Nacional de Estatística, 2002). In total, the sample comprised $2.1 \%$ of the older adults from ARM.

Participation was voluntary and participants were recruited through direct contacts carried out by the principal investigator in day-care centers, nursing homes, cultural and sport clubs and associations, and residential and public places (e.g., open markets, municipal gardens, and churches). The study was also advertised in the daily newspaper and on radio and television.

All participants filled out three questionnaires related to health status and readiness for physical activity: FallProof Health and Activity Questionnaire (Rose, 2003), MOS 36-Item Short-Form Health Survey (Ware \& Sherbourne, 1992), and Physical Activity Readiness Questionnaire (PAR-Q; Canadian Society for Exercise Physiology, 2002).

The study was approved by the Scientific Commission of the Department of Physical Education and Sports of the University of Madeira, the Regional Secretary of Education and Culture, and the Regional Secretary of Social Affairs. All participants were informed about the nature and purposes of the study, and written informed consent was obtained from each.

\section{Protocols}

Functional Fitness and Somatic Characteristics. Functional fitness was assessed with the Senior Fitness Test (SFT; Rikli \& Jones, 2001). The SFT battery was developed in the United States and was previously applied in the Autonomous Region of Azores (Santos et al., 2008). The test battery includes five components (muscle strength, aerobic endurance, flexibility, agility/dynamic balance, and body-mass index [BMI]) and seven tests (chair stand, arm curl, 6-min walk, 2-min step test, chair sit-and-reach, back scratch, and 8-ft up-and-go). Height and weight were also measured. In this study, aerobic endurance was assessed via the 6-minwalk test. A detailed description of the evaluation procedures, namely, equipment, procedures, scoring, and safety precautions, can be found in the SFT manual (Rikli \& Jones, 2001). Validity estimates for each of the functional-fitness tests have been reported previously by Rikli and Jones (1999a).

Anthropometry was collected by trained assessors. Height was recorded to the last completed unit ( $\mathrm{mm}$ ) with a portable stadiometer (Siber-Hegner, GPM). Participants stood upright with the heels, buttocks, and scapulae in contact with the backboard, feet together and the head positioned in the Frankfurt plane. Body mass was measured on a balance-beam scale accurate to $0.1 \mathrm{~kg}$ (Seca Optima 760, Germany) while the participant sat upright and still. For a detailed description of the anthropometric measurements, see Claessens, Vanden Eynde, Renson, and Van Gerven (1990).

Physical Activity. The Baecke questionnaire (Baecke, Burema, \& Frijters, 1982) was the primary instrument used to assess physical activity level in the current research. The questionnaire is composed of 16 questions, 8 related to physical activity at work and 8 related to physical activity in sports and leisure time. Item 
9 is about regular and systematic practice of sports and is formulated in terms of intensity, time, and proportion. Item responses are quantified on a Likert scale ranging from never to always or very often. This questionnaire is operationalized through three indexes: work, sport, and leisure time. Members of the field team administered the questionnaire during face-to-face interviews.

Preparation of the Field Team and Pilot Study. All assessments were conducted in the Laboratory of Human Physical Growth and Motor Development at the University of Madeira. To maximize the consistency of the assessment procedures, training sessions were conducted with five graduate students in physical education and sport, one in nursing, and three in senior education. First, a theoretical explanation of the protocols and tests to be performed was provided to all research and field-team members. Second, functional-fitness tests and questionnaires were self-administered among team members during preliminary training ( 5 days, two training sessions per day with a duration of $2 \mathrm{hr}$ ). Third, two training sessions were conducted with a group of older adults who participated in a regular physical activity program. The purposes of these additional training sessions were to test the field team in the administration of all measurements and to calculate the mean time it took to perform a complete evaluation.

The preparation of the field team and procedures was completed with a pilot study of 50 older adults, age 60-79 years. The functional-fitness tests were administered according to the instructions of the authors of the SFT (Rikli \& Jones, 2001). Test-retest correlations coefficients are presented in Table 2 . The values of the test-retest correlation $(R)$ for functional-fitness tests are .75-.90, indicating acceptable levels of reliability according to the cutoff point of .70 suggested by Safrit (1990). Intraclass correlation coefficients for height and body mass are also high (.99). For physical activity, intraclass correlation coefficients were calculated to determine the test-retest reliability of the Baecke questionnaire in a pilot study involving 32 men and 59 women (age $68.3 \pm 7.6$ years). Over an interval of 1 week, correlations were $.83, .85$, and .85 for the work, sport, and leisure-time indices, respectively. This is comparable to the reliability coefficients obtained by Baecke et al. (1982) in a sample of Dutch adult men and women $(.88, .81$, and .74) and in a more recent study by Ono et al. (2007) of middle-aged women (.84, .83, and .78). However, our correlations were higher for leisure-time index than those reported by either Baecke et al. or Ono et al., .74 and .78, respectively. The validity of the

\section{Table 2 Changes From Test to Retest: Pilot Study}

\begin{tabular}{lccccc}
\hline Functional-fitness test & $\boldsymbol{n}$ & Test, $\boldsymbol{M}(\mathbf{S D})$ & Retest, $\boldsymbol{M}(\boldsymbol{S D})$ & $\boldsymbol{R}$ & $\mathbf{9 5 \%} \mathbf{C l}$ \\
\hline Chair stand, $n$ & 49 & $17.6(4.6)$ & $18.5(3.9)$ & .772 & $.596-.871$ \\
Arm curl, $n$ & 50 & $22.8(5.7)$ & $24.2(5.3)$ & .895 & $.815-.940$ \\
6-min walk, m & 49 & $567.4(116.9)$ & $575.6(95.1)$ & .784 & $.617-.878$ \\
Chair sit-and-reach, cm & 47 & $9.8(10.7)$ & $7.9(9.2)$ & .903 & $.825-.946$ \\
Back scratch, cm & 50 & $-7.1(10.6)$ & $-6.1(10.5)$ & .749 & $.558-.858$ \\
8-ft up-and-go, s & 50 & $4.9(1.0)$ & $4.8(1.1)$ & .858 & $.749-.919$ \\
Height, cm & 50 & $155.9(6.5)$ & $155.9(6.5)$ & .999 & $.998-.999$ \\
Body mass, kg & 50 & $74.3(12.2)$ & $74.7(12.2)$ & .999 & $.999-1.000$ \\
\hline
\end{tabular}


Baecke questionnaire has also been established by Ono et al. for this population against the more objective measure of movement counts using digital pedometry and uniaxial accelerometry (Lifecorder, Suzuken Co., Nagoya, Japan); correlations ranged from .30 to .49 for the three dimensions of physical activity assessed with the Baecke questionnaire in this study.

Statistical and Smoothing Procedures. Data were entered once in the computer by two different people-each person entered the data once, in a total of two entries - and cross-referenced in specific software to detect input errors. Variables are presented as basic descriptive measures: mean, standard deviation, and percentile. Exploratory analysis of data took place through the usual procedures for identifying outliers and normality of distributions. Test-retest reliability was estimated from the intraclass correlation coefficient. For the primary purpose of the study, a smoothing procedure for the 10th-, 25th-, 50th-, 75th-, and 90th-percentile curves was carried out in two steps. First, the raw values were smoothed by eye with a graphical fitting procedure and using a flexible ruler. Second, Adobe Illustrator CS5 software tools (Adobe Systems Inc.) were used to fit the final curves. A similar procedure was used by Tanner et al. (1983) and Freitas et al. (2004). One advantage in fitting graphically rather than by computer is that we were not constrained by having to fit a preselected curve of known form (Tanner, Whitehouse, \& Takaishi, 1966). Furthermore, Cole (1998) suggests that there is much to commend this method, as the necessary tradeoff between goodness of fit and smoothness is provided by the investigator. For the second and third purposes of the study, a two-way ANOVA was used to look at the individual and joint effects of sex and age or physical activity groups on each functional-fitness test. Participants were classified separately by age cohort and sex into tertiles of high (active), moderate (average active), or low (nonactive) physical activity levels based on their responses to this questionnaire. Tukey's post hoc test was used to detect differences among age or activity groups. One-way ANCOVA was used to explore differences in functional-fitness tests between physical activity groups when controlling for height, weight, BMI, and chronological age. The calculations were made in STATA 11 (Stata Statistical Software) and SPSS 17 (Statistical Package for Social Sciences). Statistical significance was maintained at $p<.05$.

\section{Results}

Table 3 presents the scores for each functional-fitness test by sex and age group. Results from the two-way ANOVA revealed no significant interaction effects (Sex $\times$ Age Group) for any of the tests; that is, there was no significant difference in the effect of age on functional-fitness tests for men and women. A main effect of sex, with all age groups collapsed, was found for the chair stand, $F(1,794)=14.04$, partial $\eta^{2} .017$; 6-min walk, $F(1,794)=72.71$, partial $\eta^{2} .084$; chair sit-and-reach, $F(1,794)=25.56$, partial $\eta^{2} .031$; back scratch, $F(1,794)=111.05$, partial $\eta^{2} .123$; and 8-ft up-and-go, $F(1,794)=13.96$, partial $\eta^{2} .017$. Men scored significantly better than women for the chair stand, 8-ft up-and-go, and 6-min walk. Women scored significantly better than men for the chair sit-and-reach and back scratch. There was no significant main effect of sex for the arm-curl test, $F(1,749)=37.94$, $p=.127$, partial $\eta^{2} .003$. Significant main effects for age group were found for the 


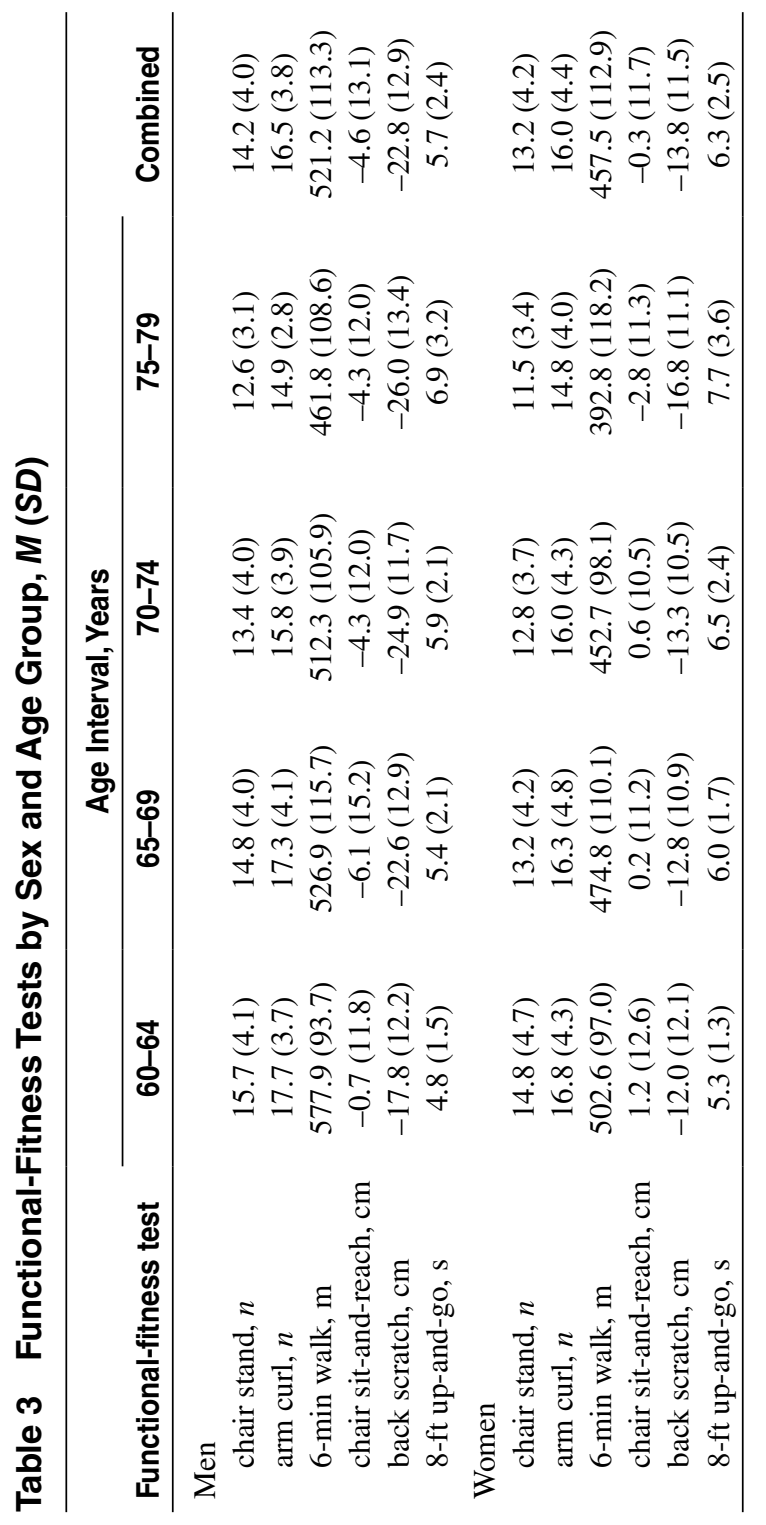


chair stand, $F(3,794)=22.95$, partial $\eta^{2} .080$; arm curl, $F(3,794)=13.27$, partial $\eta^{2} .048$; 6-min walk, $F(13,794)=37.90$, partial $\eta^{2} .125$; chair sit-and-reach, $F(3$, $794)=5.97$, partial $\eta^{2} .022$; back scratch, $F(3,794)=8.84$, partial $\eta^{2} .032$; and 8 -ft up-and-go, $F(3,794)=33.55$, partial $\eta^{2} .112$.

In general, participants' performance declined with advancing age. Post hoc comparisons using Tukey's honestly significant difference test indicated that the mean score for Group 1 (60-64 years, $M=15.23, S D=4.45)$ was significantly different from Groups 2 (65-69 years, $M=13.99, S D=4.19), 3(70-74$ years, $M$ $=13.13, S D=3.87)$, and $4(75-79$ years, $M=12.03, S D=3.29)$ in the chair-stand test. Group 2 also scored significantly better than Group 4. For the arm-curl test, Group $1(M=17.23, S D=4.02)$ performed better than Groups $3(M=15.93, S D$ $=4.09)$ and $4(M=14.85, S D=3.43)$. Groups $2(M=16.81, S D=4.46)$ and 3 scored better than Group 4. For the 6-min-walk test, the mean score for Group 1 $(M=540.41, S D=102.36)$ was significantly different from Groups $2(M=499.72$, $S D=115.51), 3(M=483.81, S D=106.25)$, and $4(M=427.12, S D=118.41)$. A better performance of Group 3 was also observed than that of Group 4. For the flexibility tests, a lower negative score corresponds to a worse score. In the chair sit-and-reach test, Group $1(M=-0.02, S D=12.69)$ performed better than Group $4(M=-5.10, S D=11.54)$. No significant differences were found between the means of the remaining pairs of age groups. For the back-scratch test, Group $1(M$ $=-15.31, S D=12.91)$ was more proficient than Groups $3(M=-19.37, S D=12.59)$ and $4(M=-21.37, S D=13.10)$. Group $2(M=-17.51, S D=12.84)$ performed better than Group 4. The unit of measurement in the 8-ft up-and-go test is seconds, so the shorter the time interval the higher the participant's performance. Group 1 $(M=5.00, S D=1.47)$ performed significantly better than Groups $3(M=6.16, S D$ $=2.27)$ and $4(M=7.31, S D=3.40)$. Groups $2(M=5.72, S D=1.92)$ and 3 also performed better than Group 4 .

\section{Developmental Growth Curves}

Age and sex differences described earlier are clearly seen in the developmental curves (P50 values) for the different tests (Figures 1-6). For muscle strength (chairstand and arm-curl tests), interindividual variability - the absolute range between the 10th and 90th percentiles - is larger at 60-64 than at 75-79 years of age in men and women, respectively (Figures 1 and 2). The variability for aerobic endurance, as measured by the 6-min-walk test, is higher in the oldest (75-79) than in the youngest age (60-64) cohort. Women demonstrate somewhat greater variability on this test than men at 60-64 (219 m vs. $210 \mathrm{~m})$ and 75-79 years of age (340.2 m vs. 277 m; Figure 3). For lower body flexibility (sit-and-reach test), the range of variability is larger in the older (75-79 years of age) than younger age cohorts, whereas the performance on this test was much more consistent and less variable across age cohorts for the women: $33.84 \mathrm{~cm}$ (60-64 years) and $27.12 \mathrm{~cm}$ (75-79 years; Figure 4). For upper body flexibility (back-scratch test), variability increases with age for men (from $25 \mathrm{~cm}$ to $30.4 \mathrm{~cm}$ between the youngest and oldest cohorts) and decreases for women (from $21.85 \mathrm{~cm}$ to $17.5 \mathrm{~cm}$; Figure 5). The performance in agility/dynamic balance (8-ft up-and-go) is shown in Figure 6. Interindividual variability increases with age. 


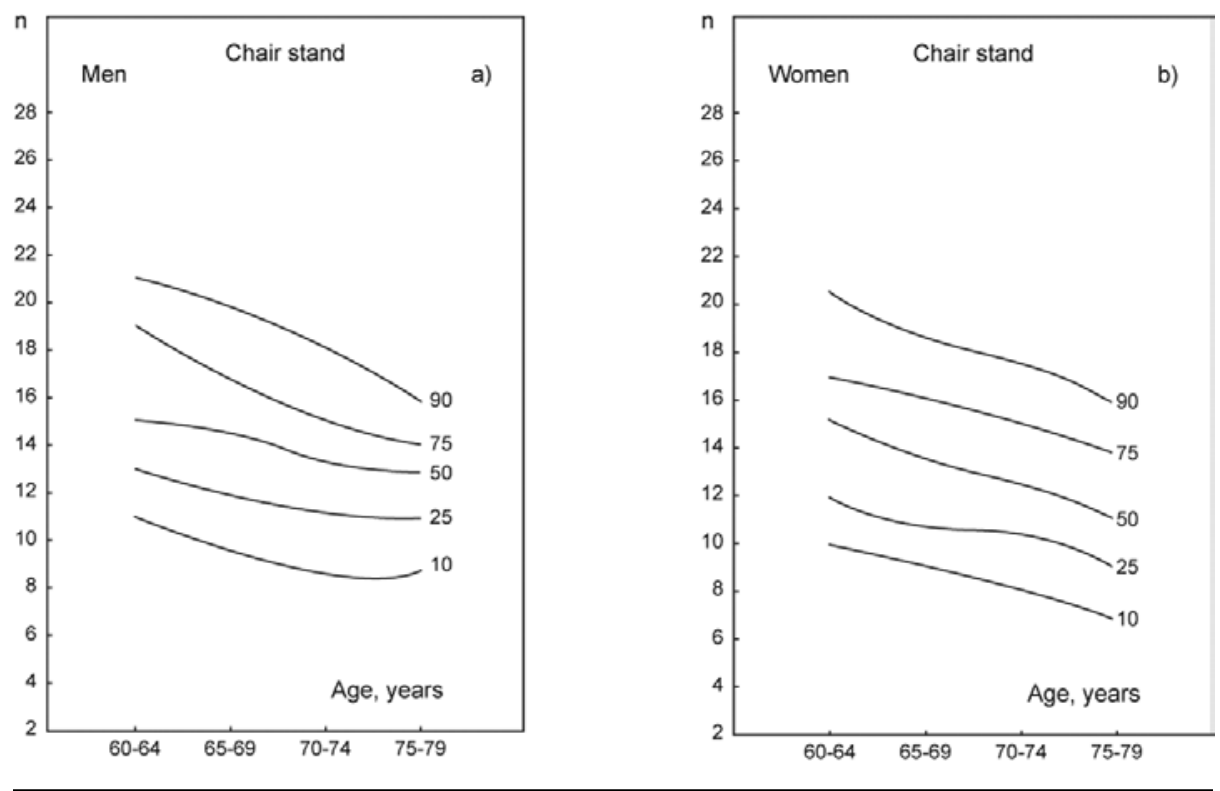

Figure 1 - Percentile distribution of Madeira older adults: chair stand.
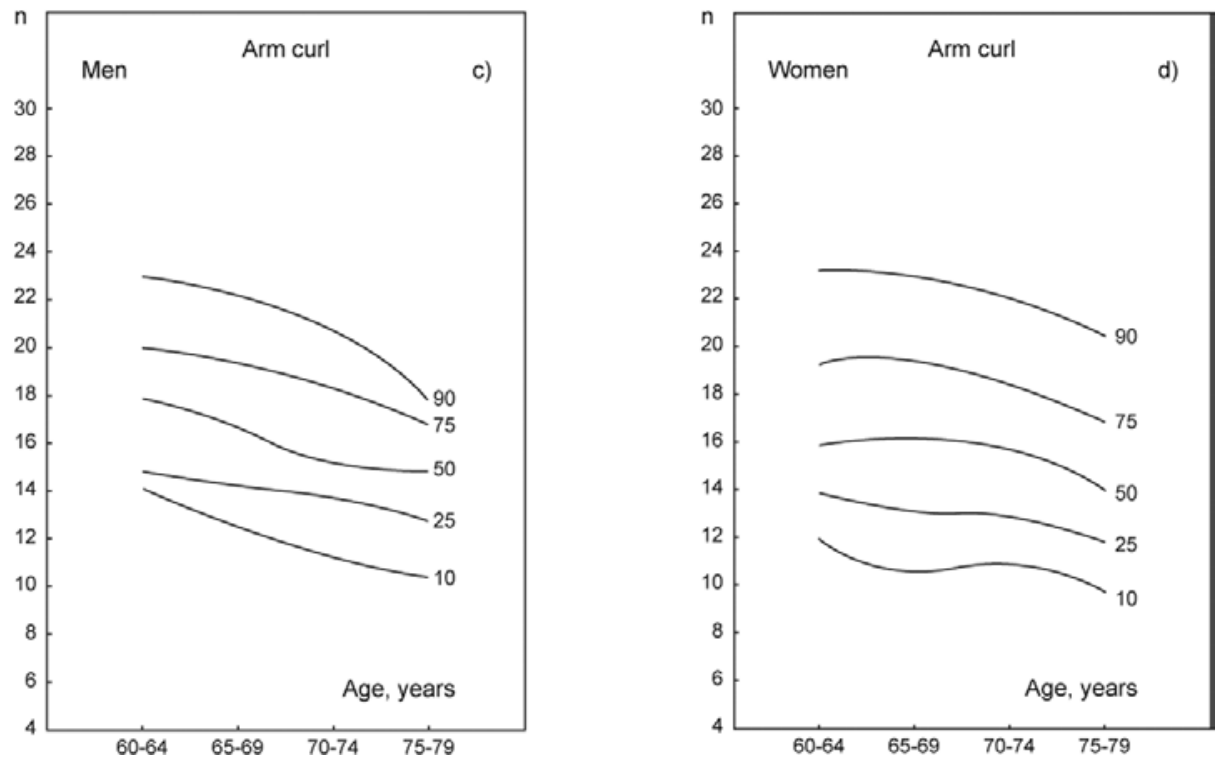

Figure 2 - Percentile distribution of Madeira older adults: arm curl. 

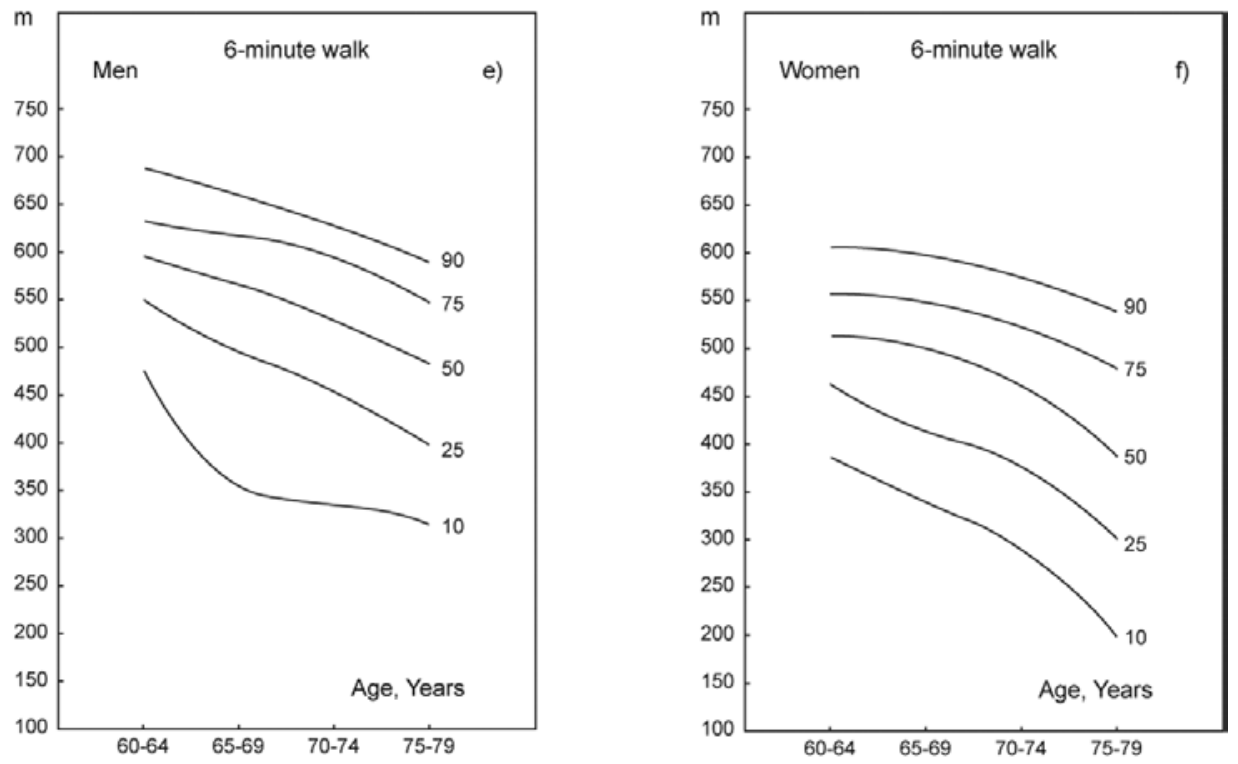

Figure 3 - Percentile distribution of Madeira older adults: 6-min walk.
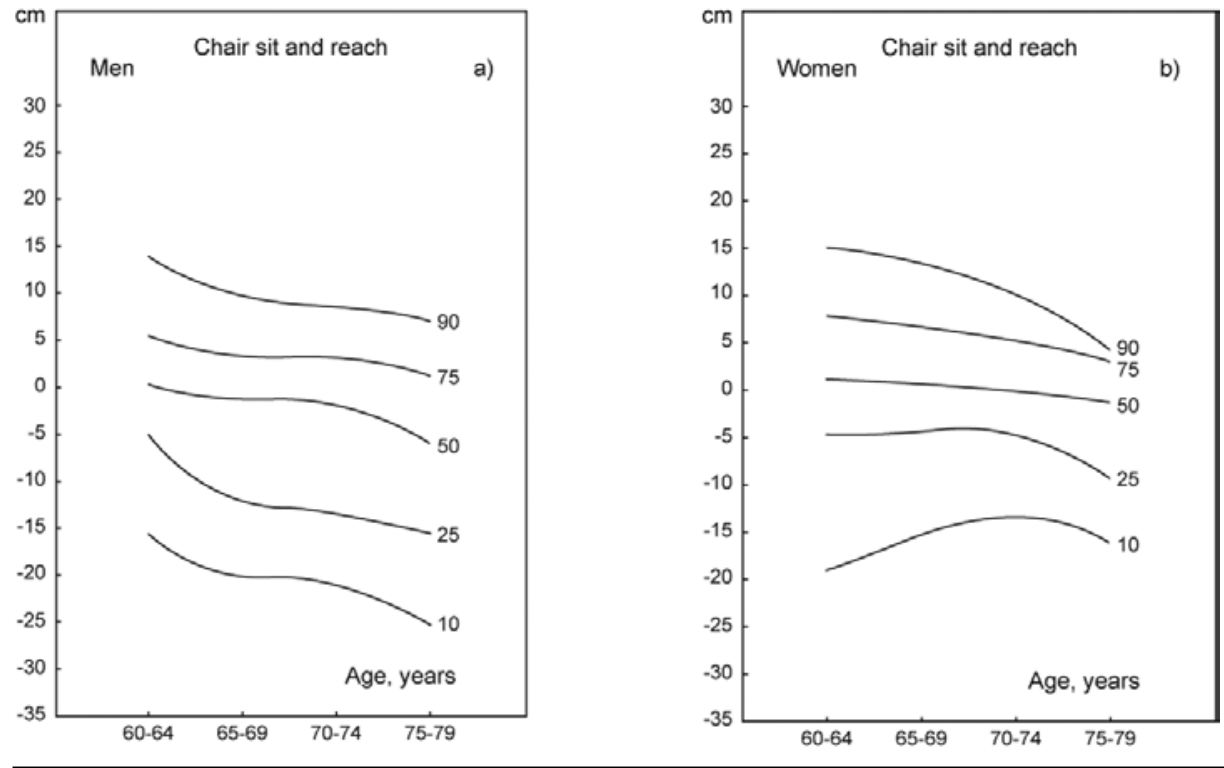

Figure 4 - Percentile distribution of Madeira older adults: chair sit-and-reach. 

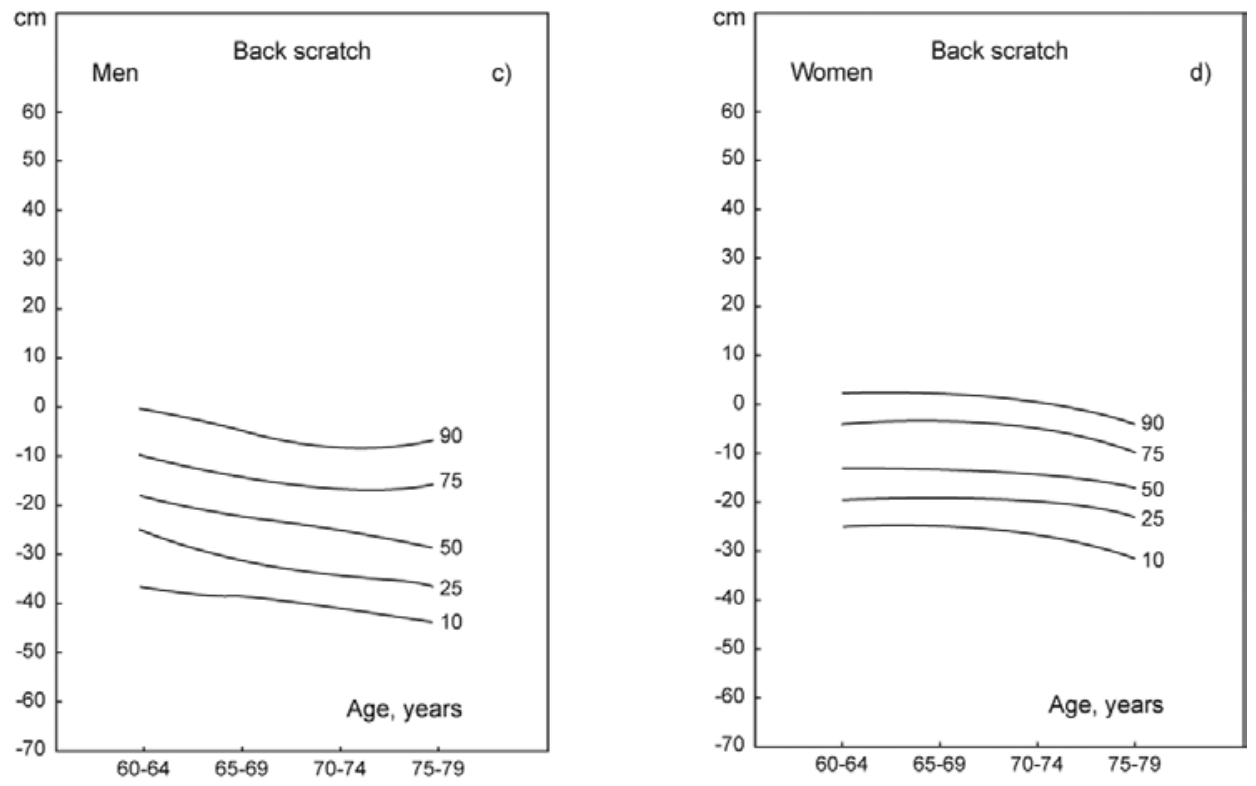

Figure 5 - Percentile distribution of Madeira older adults: back scratch.
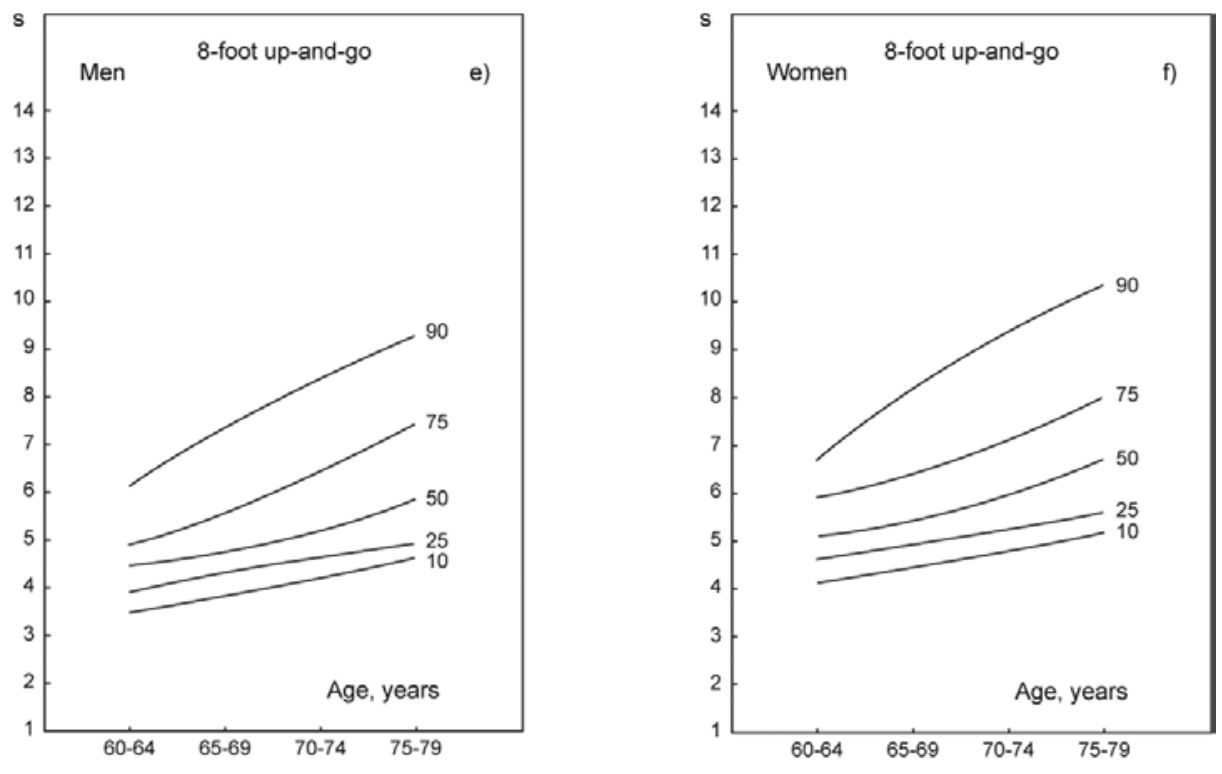

Figure 6 - Percentile distribution of Madeira older adults: 8-ft up-and-go. 


\section{Physical Activity-Associated Variation in Functional Fitness}

Means and standard deviations for the functional-fitness tests, by level of physical activity and sex, are presented in Table 4. Results from the two-way ANOVA indicated an interaction effect (Sex $\times$ Physical Activity) for the 6-min-walk test, $F(2,796)=6.14$; however, the effect size was small (partial $\eta^{2} .015$ ). A main effect of sex on functional-fitness tests has been previously reported. The impact of physical activity on functional fitness was significant for chair stand, $F(2,796)$ $=46.17$, partial $\eta^{2} .104$; arm curl, $F(2,796)=35.85$, partial $\eta^{2} .083 ; 6$-min walk, $F(2,796)=55.47$, partial $\eta^{2} .122$; chair sit-and-reach, $F(2,796)=16.66, \eta^{2} .040$; back scratch, $F(2,796)=9.45$, partial $\eta^{2} .023$; and 8-ft up-and-go, $F(2,796)=$ 45.92, partial $\eta^{2} .103$.

Post hoc comparisons revealed that participants with high $(M=15.31, S D=$ 4.07) and moderate $(M=13.69, S D=3.68)$ physical activity (PA) levels performed significantly better than their peers with low $(M=12.09, S D=4.05)$ PA levels in the chair-stand test. The mean score for participants with high $(M=17.84, S D=$ 4.06) and moderate $(M=16.06, S D=4.05)$ PA levels was statistically different from participants with low $(M=14.99, S D=3.77)$ PA levels in the arm-curl test. This relationship was also observed between participants with high and moderate PA levels. For the 6-min-walk test, the high $(M=537.50, S D=95.39)$ PA group performed significantly better than the moderate $(M=492.88, S D=98.08)$ and low $(M=442.84, S D=133.38)$ PA groups. Similarly, the moderate PA group scored significantly better than low PA group. In the chair sit-and-reach test, participants

Table 4 Functional-Fitness Tests by Sex and Physical Activity Level

\begin{tabular}{|c|c|c|c|c|c|c|}
\hline \multirow[b]{3}{*}{ Functional-fitness test } & \multicolumn{6}{|c|}{ Physical Activity Level } \\
\hline & \multicolumn{2}{|r|}{ Nonactive } & \multicolumn{2}{|c|}{ Average Active } & \multicolumn{2}{|r|}{ Active } \\
\hline & $n$ & Measure & $n$ & Measure & $n$ & Measure \\
\hline \multicolumn{7}{|l|}{ Men } \\
\hline chair stand, $n$ & 147 & $12.9(3.7)$ & 122 & $14.2(3.8)$ & 132 & $15.5(4.2)$ \\
\hline arm curl, $n$ & 147 & $15.6(3.6)$ & 122 & $16.2(3.6)$ & 132 & $17.7(4.0)$ \\
\hline 6-min walk, $\mathrm{m}$ & 147 & $484.7(129.4)$ & 122 & $534.0(92.9)$ & 132 & $550.0(100.5)$ \\
\hline chair sit-and-reach, cm & 147 & $-7.1(12.9)$ & 122 & $-2.9(11.6)$ & 132 & $-3.6(14.1)$ \\
\hline back scratch, cm & 147 & $-24.2(12.7)$ & 122 & $-23.4(13.3)$ & 132 & $-20.8(12.7)$ \\
\hline 8-ft up-and-go, s & 147 & $6.6(3.3)$ & 122 & $5.4(1.4)$ & 132 & $5.0(1.6)$ \\
\hline \multicolumn{7}{|l|}{ Women } \\
\hline chair stand, $n$ & 135 & $11.2(4.2)$ & 146 & $13.2(3.5)$ & 120 & $13.1(4.2)$ \\
\hline arm curl, $n$ & 135 & $14.3(3.9)$ & 146 & $16.0(4.4)$ & 120 & $18.0(4.1)$ \\
\hline 6-min walk, m & 135 & $397.3(122.6)$ & 146 & $458.6(88.9)$ & 120 & $523.8(87.8)$ \\
\hline chair sit-and-reach, cm & 135 & $-4.4(13.5)$ & 146 & $0.4(10.2)$ & 120 & $3.6(9.9)$ \\
\hline back scratch, $\mathrm{cm}$ & 135 & $-17.1(11.4)$ & 146 & $-12.8(11.8)$ & 120 & $-11.4(10.5)$ \\
\hline 8-ft up-and-go, s & 135 & $7.5(3.5)$ & 146 & $6.0(1.8)$ & 120 & $5.3(1.1)$ \\
\hline
\end{tabular}


with high $(M=-0.17, S D=12.78)$ and moderate $(M=-1.11, S D=10.96)$ PA levels performed significantly better than their peers with low $(M=-5.79, S D=13.22)$ PA levels. For the back-scratch test, the high PA group $(M=-16.31, S D=12.59)$ scored significantly better than the moderate $(M=-17.61, S D=13.52)$ and low $(M$ $=-20.78, S D=12.56)$ PA groups. Finally, for the 8-ft up-and-go test, participants with high $(M=5.17, S D=1.38)$ and moderate $(M=5.75, S D=1.62)$ PA levels scored significantly better than their peers with low $(M=7.03, S D=3.41)$ PA levels. This direction of results was also presented by high and moderate PA levels.

\section{Discussion}

Older adults from ARM showed, on average, lower functional fitness than younger seniors, and men were fitter than women, with the exception of flexibility. The higher active participants performed better than their medium and lower active peers.

It is difficult to compare the current results with other studies due to differences among participants (i.e., birth cohort, race/ethnicity, lifestyle, physical activity levels, and culture) and methodological aspects related to sampling (i.e., active vs. passive recruitment, stratified vs. nonstratified, etc.). However, a decline with age in muscle strength/endurance, aerobic endurance, flexibility, and agility/balance has been observed in Brazilian (Krause et al., 2009), Japanese (Demura et al., 2003), and North American older adults (Rikli \& Jones, 1999b).

The difference between sexes observed in the Madeira sample parallels other studies. In Japanese older adults (60-89 years), Demura et al. (2003) observed that men had better performance than women in muscle strength and aerobic capacity. In contrast, women were more flexible than men. In a North American sample, Rikli and Jones (1999b) found that men outperformed women in strength, aerobic endurance, and agility/balance; women scored better in flexibility tests. The higher values for men in muscle strength can be explained by higher gains during puberty (Malina, Bouchard, \& Bar-Or, 2004) and smaller losses from 65 years onward compared with women (Shephard, 2002). Moreover, the superiority of women in flexibility seems to result from differences in tissue architecture and morphology of the skeletal tissue (Holland, Tanaka, Shigematsu, \& Nakagaichi, 2002).

Comparing the Portuguese (current study) and U.S. (Rikli \& Jones, 1999b) samples, the Portuguese older adults showed poorer performances in the chair-stand, arm-curl, 6-min-walk, chair sit-and-reach, back-scratch, and 8-ft up-and-go tests. Results for the two studies were more similar for the chair stand (women), 6-min walk (women), and 8-ft up-and-go (men and women). There was a large difference in back-scratch test performance between Portuguese and North American participants, favoring the North Americans.

The positive association between physical activity and functional fitness in our Madeira elderly was consistent with other studies. In older Dutch adults, Voorrips, Lemmink, Heuvelen, Bult, and Staveren (1993) observed higher mean values for flexibility and walking in active than in nonactive participants. In Canada, Sawatzky and Naimark (2002) found that older women, especially those with moderate or vigorous physical activity, have a healthier cardiovascular profile than their less active peers. Similarly, Tanaka, Shigematsu, Nakagaichi, Kim, and Takeshima (2000) found a positive association between physical activity and functional fitness in older Japanese adults. In addition, in Finland, active participants (75 years 
or older) performed better than their sedentary peers (Laukkanen, Kauppinen, \& Heikkinen, 1998). In the scope of the Baltimore Longitudinal Study of Aging, Fleg et al. (2005) found that higher average values of physical activity were accompanied by higher levels of $\mathrm{VO}_{2 \max }$, and this relationship was maintained across all age intervals. In the Autonomous Region of Azores, Santos et al. (2008) found that active men performed better than their nonactive peers on the chair-stand, 8 - $\mathrm{ft}$ up-and-go, chair sit-and-reach, and back-scratch tests.

The positive association between physical activity and the maintenance of functional fitness could play an important role in the functionality, mobility, autonomy, health, and welfare of elderly adults (Fleg et al., 2005). For example, high values of functional fitness, especially aerobic endurance, have been associated with a lower risk of mortality from all causes and cardiovascular events (Kodama et al., 2009; Sui et al., 2007). Likewise, high levels of physical activity in older adults are associated with a more favorable profile of cardiovascular biomarkers (Mora, Lee, Buring, \& Ridker, 2006) and a lower risk for Alzheimer's disease (Scarmeas et al., 2009) and diabetes mellitus (Weinstein et al., 2004).

Data from the Cardiovascular Health Study (Hirsch et al., 2010) also suggest gains in survival (years of life) and years of healthy life (the number of years during which a participant reported being in good to excellent health vs. fair or poor health or dead in the following 11 years) in older adults who are active. Compared with sedentary older adults, the most active men ( 75 years and older) had 1.49 more years of healthy life $(95 \%$ CI: $0.79,2.19)$, and the most active women ( 75 years and older) had 1.06 more years of healthy life (95\% CI: 0.44, 1.68). Similar results were found by Baker, Meisner, Logan, Kungl, and Weir (2009) in older Canadian adults. Active participants were more than twice as likely to be rated as aging successfully, even after removing variance associated with demographic covariates.

Although there was a clear positive association between functional fitness and physical activity in this study, the validity of this association may be affected by the questionnaire approach that was used to quantify physical activity levels. In older adults, there is no consensus regarding the most valid and accurate way of assessing physical activity, and there is little information about how well older people are able to evaluate their activity levels (Sihvonen, Rantanen, \& Heikkinen, 1998). To minimize problems of perception, encoding, storage, and retrieval of information, the questionnaire in our study was administered during a face-to-face interview. In addition, size, body composition, and age can mask this association (Kostic, Uzunovic, Pantelic, \& Duraskovic, 2011; Kyle et al., 2001; Tuna, Edeer, Malkoc, \& Aksakoglu, 2009; Zamboni et al., 1999). To explore the variation in functional fitness according to physical activity levels, height, weight, BMI, and age were used as covariates. Differences in functional-fitness tests according to physical activity levels remained statistically significant. An exception was observed for the back-scratch test in men. After adjusting for covariates, there was no significant difference between the three physical activity groups on the back-scratch test.

Our findings may also be limited by two other factors, namely, sample characteristics and study design. Even though our study had a reasonable sample size and extended to the eighth decade, data from even older adults ( 80 years and over) are lacking. In addition, we used media-announcement (passively recruited) and personal-contact (actively recruited) sampling procedures. Heuvelen, Stevens, and Kempen (2002) observed that the performance of a passively recruited sample 
was significantly better on several functional-fitness tests than that of an actively recruited sample. As for the latter potential limitation, the cross-sectional design is inadequate to capture the temporal relations that occur throughout life, and this approach precludes inference of causality between physical activity and functional ability.

Our research approach also has substantive strength. First, the sample included older adults from Madeira and Porto Santo Islands and was the first study to investigate these relationships in this population. Second, the data were collected by nine well-trained team members graduated in physical education and sports, nursing, and senior education, ensuring protocol consistency and minimizing data-collection errors. Third, we gathered information about physical activity and explored the variation in functional fitness according to physical activity levels: This has not been examined extensively in the elderly. Finally, the measurement protocols were first implemented in a pilot study that verified high reliabilities.

In sum, this study and its generated growth curves provide population-specific and relevant data for Portuguese older adults. Muscle strength, aerobic endurance, flexibility, and agility/dynamic balance declined over age, and men generally performed better on tests of functional fitness than women. Furthermore and notably, the active participants scored better on functional-fitness tests than their nonactive peers. Thus, any intervention in older adults should take into account the loss of functionality and differences between women and men in fitness performance. More research, particularly longitudinal studies including adults over 80 years of age, is needed in ARM for a more thorough understanding of these relations.

\section{Acknowledgments}

The authors are grateful to Bruna Gouveia, Joana Castro, and Letícia Sousa for their technical assistance in data collection and Roberta E. Rikli and C. Jessie Jones for their comments and suggestions that helped to improve the manuscript.

This manuscript was funded by a doctorate scholarship from the Portuguese Foundation for Science and Technology, reference SFRH/BD/29300/2006, and Government of Madeira-Regional Secretary of Education and Culture, Portugal.

\section{References}

American College of Sports Medicine. (2006). ACSM's guidelines for exercise testing and prescription (7th ed.). Philadelphia: Lippincott Williams \& Wilkins.

Baecke, J.A., Burema, J., \& Frijters, J.E. (1982). A short questionnaire for the measurement of habitual physical activity in epidemiological studies. The American Journal of Clinical Nutrition, 36(5), 936-942. PubMed

Baker, J., Meisner, B.A., Logan, A.J., Kungl, A-M., \& Weir, P. (2009). Physical activity and successful aging in Canadian older adults. Journal of Aging and Physical Activity, 17(2), 223-235. PubMed

Canadian Society for Exercise Physiology. (2002). Physical Activity Readiness Questionnaire-PAR-Q. Revised version. Ottawa, ON: Expert Advisory Committee of the Canadian Society for Exercise Physiology. Retrieved from www.csep.ca/forms

Carrilho, M.J., \& Gonçalves, C. (2004). Dinâmicas territoriais do envelhecimento: Análise exploratória dos resultados dos Censos 91 e 2001. Revista de Estudos Demográficos, $36,175-191$. 
Claessens, A., Vanden Eynde, B., Renson, R., \& Van Gerven, D. (1990). The description of tests and measurements. In J. Simons, G. Beunen, R. Renson, A. Claessens, B. Vanreusel, \& J. Lefevre, (Eds.), Growth and fitness of Flemish girls-The Leuven Growth Study (Vol. 3, HKP Sport Science Monograph Series, pp 21-39). Champaign, IL: Human Kinetics.

Cole, T. (1998). Creation of growth references. In S.J. Ulijaszek, F.E. Johnston, \& M.A. Preece (Eds.), The Cambridge encyclopedia of human growth and development, part one: Measurement and assessment (pp. 63-65). Cambridge, UK: Cambridge University Press.

Demura, S., Minami, M., Nagasawa, Y., Tada, N., Matsuzawa, J., \& Sato, S. (2003). Physicalfitness declines in older Japanese adults. Journal of Aging and Physical Activity, 11(1), $112-122$.

Fleg, J.L., Morrell, C.H., Bos, A.G., Brant, L.J., Talbot, L.A., Wright, J.G., \& Lakatta, E.G. (2005). Accelerated longitudinal decline of aerobic capacity in healthy older adults. Circulation, 112(5), 674-682. PubMed doi:10.1161/CIRCULATIONAHA.105.545459

Freitas, D., Maia, J., Beunen, G., Lefevre, J., Claessens, A., Marques, A., . . Malina, R. (2004). Skeletal maturity and socio-economic status in Portuguese children and youths: The Madeira Growth Study. Annals of Human Biology, 31(4), 408-420. PubMed doi: 10.1080/03014460410001713050

Heuvelen, M., Stevens, M., \& Kempen, G.I. (2002). Differences in physical-fitness test scores between actively and passively recruited older adults: Consequences for norm-based classification. Journal of Aging and Physical Activity, 10(2), 143-159.

Hirsch, C.H., Diehr, P., Newman, A.B., Gerrior, S.A., Pratt, C., Lebowitz, M.D., \& Jackson, S.A. (2010). Physical activity and years of healthy life in older adults: Results from the Cardiovascular Health Study. Journal of Aging and Physical Activity, 18(3), 313-334. PubMed

Holland, G.J., Tanaka, K., Shigematsu, R., \& Nakagaichi, M. (2002). Flexibility and physical functions of older adults: A review. Journal of Aging and Physical Activity, 10(2), 169-206.

Instituto Nacional de Estatística. (2002). Censos 2001: Resultados definitivos: XIV recenseamento geral da população: IV recenseamento geral da habitação. Lisbon, Portugal: Instituto Nacional de Estatística.

Kodama, S., Saito, K., Tanaka, S., Maki, M., Yachi, Y., Asumi, M., . . Sone, H. (2009). Cardiorespiratory fitness as a quantitative predictor of all-cause mortality and cardiovascular events in healthy men and women. A meta-analysis. Journal of the American Medical Association, 301(19), 2024-2035. PubMed doi:10.1001/jama.2009.681

Kostic, R., Uzunovic, S., Pantelic, S., \& Duraskovic, R. (2011). A comparative analysis of the indicators of the functional fitness of the elderly. Facta Universitatis, 9(2), 161-171.

Krause, M.P., Januário, R.S., Hallage, T., Haile, L., Miculis, C.P., Gama, M.P., . . Silva, S.G. (2009). A comparison of functional fitness of older Brazilian and American women. Journal of Aging and Physical Activity, 17(4), 387-397. PubMed

Kyle, U.G., Genton, L., Hans, D., Karsegard, L., Slosman, DO., \& Pichard, C. (2001). Age-related differences in fat-free mass, skeletal muscle, body cell mass and fat mass between 18 and 94 years. European Journal of Clinical Nutrition, 55(8), 663-672.

Laukkanen, P., Kauppinen, M., \& Heikkinen, E. (1998). Physical activity as a predictor of health and disability in 75- and 80-year-old men and women: A five-year longitudinal study. Journal of Aging and Physical Activity, 6(2), 141-156.

Malina, R.M., Bouchard, C., \& Bar-Or, O. (2004). Growth, maturation, and physical activity. Champaign, IL: Human Kinetics.

Mora, S., Lee, I-M., Buring, J.E., \& Ridker, P.M. (2006). Association of physical activity and body mass index with novel and traditional cardiovascular biomarkers in women. Journal of the American Medical Association, 295(12), 1412-1419. PubMed doi:10.1001/jama.295.12.1412 
Ono, R., Hirata, S., Yamada, M., Nishiyama, T., Kurosaka, M., \& Tamura, Y. (2007). Reliability and validity of the Baecke Physical Activity Questionnaire in adult women with hip disorders. BMC Musculoskeletal Disorders, 8, 61. PubMed doi:10.1186/1471-2474-8-61

Rikli, R.E., \& Jones, C.J. (1999a). Development and validation of a functional fitness test for community-residing older adults. Journal of Aging and Physical Activity, 7(2), 129-161.

Rikli, R.E., \& Jones, C.J. (1999b). Functional fitness normative scores for communityresiding older adults, ages 60-94. Journal of Aging and Physical Activity, 7(2), 162-181.

Rikli, R.E., \& Jones, C.J. (2001). Senior fitness test manual. Development and validation of a functional fitness test for community-residing older adults. Champaign, IL: Human Kinetics.

Rose, D.J. (2003). FallProof! A comprehensive balance and mobility training program. Champaign, IL: Human Kinetics.

Safrit, M. (1990). The validity and reliability of fitness tests for children: A review. Pediatric Exercise Science, 2(1), 9-28.

Santos, R., \& Moreira, P.M., Ribeiro, J.C., Carvalho, J., Santos, M.P., . . Ramos, E. (2008). Carta da condição física dos cidadãos da Região Autónoma dos Açores. Centro de Investigação em Actividade, Saúde e Lazer. Faculdade de Desporto da Universidade do Porto. Direcção Regional do Desporto da Região Autónoma dos Açores. Porto, Portugal: Multitema.

Sawatzky, J.A., \& Naimark, B.J. (2002). Physical activity and cardiovascular health in aging women: A health-promotion perspective. Journal of Aging and Physical Activity, 10(4), 396-412.

Scarmeas, N., Luchsinger, J.A., Schupf, N., Brickman, A.M., Cosentino, S., Tang, M.X., \& Stern, Y. (2009). Physical activity, diet, and risk of Alzheimer disease. Journal of the American Medical Association, 302(6), 627-637. PubMed doi:10.1001/jama.2009.1144

Shephard, R.J. (Ed.). (2002). Gender, physical activity, and aging. Boca Raton, FL: CRS Press.

Sihvonen, S., Rantanen, T., \& Heikkinen, E. (1998). Physical activity and survival in elderly people: A five-year follow-up study. Journal of Aging and Physical Activity, 6(2), 133-140.

Spirduso, W.W., Francis, K.L., \& MacRae, P.G. (2005). Physical dimensions of aging (2nd ed.). Champaign, IL: Human Kinetics.

Sui, X., LaMonte, M.J., Laditka, J.N., Hardin, J.W., Chase, N., Hooker, S.P., \& Blair, S.N. (2007). Cardiorespiratory fitness and adiposity as mortality predictors in older adults. Journal of the American Medical Association, 298(21), 2507-2516. PubMed doi:10.1001/jama.298.21.2507

Tanaka, K., Shigematsu, R., Nakagaichi, M., Kim, H., \& Takeshima, N. (2000). The relationship between functional fitness and coronary heart disease risk factors in older Japanese adults. Journal of Aging and Physical Activity, 8(2), 162-174.

Tanner, J.M., Whitehouse, R.H., Cameron, N., Marshall, W.A., Healy, M.J., \& Goldstein, H. (1983). Assessment of skeletal maturity and prediction of adult height (TW2 method) (2nd ed.). London: Academic Press.

Tanner, J.M., Whitehouse, R.H., \& Takaishi, M. (1966). Standards from birth to maturity for height, weight, height velocity, and weight velocity: British children, I965. Part I. Archives of Disease in Childhood, 41(219), 454-471. PubMed doi:10.1136/ adc. 41.219 .454

Taylor, A.W., \& Johnson, M.J. (Eds.). (2008). Physiology of exercise and healthy aging. Champaign, IL: Human Kinetics.

Tuna, H.D., Edeer, A.O., Malkoc, M., \& Aksakoglu, G. (2009). Effect of age and physical activity level on functional fitness in older adults. European Review of Aging and Physical Activity, 6(2), 99-106. doi:10.1007/s11556-009-0051-z 
Voorrips, L.E., Lemmink, K.A., Heuvelen, M.J., Bult, P., \& Staveren, W.A. (1993). The physical condition of elderly women differing in habitual physical activity. Medicine and Science in Sports and Exercise, 25(10), 1152-1157. PubMed

Ware, J.E., \& Sherbourne, C.D. (1992). The MOS 36-Item Short-Form health survey (SF-36). I. Conceptual framework and item selection. Medical Care, 30(6), 473-483. PubMed doi:10.1097/00005650-199206000-00002

Weinstein, A.R., Sesso, H.D., Lee, I.M., Cook, N.R., Manson, J.E., Buring, J.E., \& Gaziano, J.M. (2004). Relationship of physical activity vs body mass index with Type 2 diabetes in women. Journal of the American Medical Association, 292(10), 1188-1194. PubMed doi:10.1001/jama.292.10.1188

World Health Organization. (2011). World health statistics 2011. Retrieved from http:// www.who.int/whosis/whostat/EN_WHS2011_Full.pdf

Zamboni, M., Turcato, E., Santana, H., Maggi, S., Harris, T.B., Pietrobelli, A., . . Bosello, O. (1999). The relationship between body composition and physical performance in older women. Journal of the American Geriatrics Society, 47(12), 1403-1408. PubMed 